\title{
Notas sobre siete especies de palmeras (Arecaceae) de bosque nublado, presentes en la vertiente noreste de la serranía de Perijá, estado Zulia, Venezuela
}

\section{Notes on seven species of cloud forest palms (Arecaceae) present in the northeast side of the serranía de Perijá, Zulia State, Venezuela}

\author{
Juan C. Arias ${ }^{1}$, Fred W. Stauffer ${ }^{2}$
}

\begin{abstract}
Resumen
Se presentan observaciones sobre siete especies de palmeras (familia Arecaceae) de bosque nublado, presentes en la vertiente noreste de la serranía de Perijá, estado Zulia, Venezuela. Los taxones descritos pertenecen a los géneros Chamaedorea Willd., Geonoma Willd., Hyospathe Mart., Prestoea Hook. f. y Wettinia Poepp. ex Endl., entre los que se destacan como novedades para el estado Zulia las especies Chamaedorea linearis y Geonoma undata, cuyas poblaciones se encontrarían seriamente amenazadas. Se realizan comentarios sobre el hábitat, distribución, amenazas y estado de conservación de estas poblaciones.
\end{abstract}

Palabras clave: Arecaceae; serranía de Perijá; Venezuela.

\begin{abstract}
We present observations on seven palm species (family Arecaceae) from cloud forest, present in the northeastern slope of serranía de Perijá, Zulia state, Venezuela. The described taxa belong to the genera Chamaedorea Willd., Geonoma Willd., Hyospathe Mart., Prestoea Hook. f. and Wettinia Poepp. ex Endl., among them the species Chamaedorea linearis and Geonoma undata are novelties for Zulia state and their populations might be in serious threat. Comments are provided on habitat, distribution, threats to these populations and their possible conservation status.
\end{abstract}

Keywords: Arecaceae; serranía de Perijá; Venezuela.

\section{Introducción}

A nivel de familias de plantas vasculares, las palmas (familia Arecaceae) poseen la mayor diversidad morfológica dentro de las Liliópsidas (Monocotiledóneas) presentando diferentes formas de crecimiento y una importante riqueza en especies (Uhl y Dransfield 1987). La familia de las palmas incluye 189 géneros y unas 2360 especies a nivel mundial, distribuidas principalmente en la región tropical, con algunas especies que se extienden hacia las áreas subtropicales (Dransfield et al. 2008).

Las palmas suelen presentar troncos estipitados y hojas pinnadas (pinnatisectas) o palmeadas (palmatisectas) que forman una corona al final del tallo, características que le confieren a las Arecaceae el título de plantas emblemáticas de los trópicos. Estas plantas presentan tallos gruesos o delgados, con superficie lisa, anillada o con espinas bien pronunciadas; sus inflorescencias presentan flores trímeras, hermafroditas o unisexuales. Los frutos son bayas o drupas con pericarpio carnoso o fibroso, siendo aprovechados y considerados como recursos promisorios para las sociedades humanas en muchas localidades alrededor del mundo (Balslev y Barfod 1987, Balick y Beck 1990, Borchsenius y Moraes 2006).

\footnotetext{
Universidad del Zulia, Facultad Experimental de Ciencias. Apartado postal 526. Maracaibo 4011, estado Zulia, Venezuela. e-mail: jcariasj@gmail.com

2 Conservatoire et Jardin botaniques de la Ville de Genève. Ch. de 1'Impératrice 1, Case Postale 60, CH-1292. Chambésy, Genève, Suiza. e-mail: Fred.Stauffer@ville-ge.ch

Fecha recepción: Noviembre 15, 2012

Fecha aprobación: Diciembre 26, 2012
} 
Las arecáceas ofrecen recursos de hábitat y disponibilidad de alimentos a muchas especies animales y vegetales al proveer de sustrato para diversos organismos epífitos y producir flores y frutos que son aprovechados por animales nectarívoros y frugívoros (Cabrera y Wallace 2007). Suelen ser abundantes en términos de especies e individuos y se encuentran en todos los estratos de la comunidad vegetal (Scariot 1999) constituyendo un elemento conspicuo e importante en la estructura y ecología de los bosques tropicales (Balslev et al. 2008).

En las regiones tropicales las palmas crecen en los más variados climas, siendo más frecuentes en el bosque tropical húmedo (Hoyos y Braun 2001). En Los Andes y por encima de $1000 \mathrm{~m}$ de altitud se ha registrado un total de 24 (37\%) géneros y 121 (15\%) especies de palmeras, de la riqueza total reportada para el continente americano (Borchsenius y Moraes 2006, Pintaud et al. 2008). La mayoría de los géneros representados en Los Andes tienen su centro de diversidad fuera de esa región, pero siete géneros poseen más de $2 / 3$ de sus especies en la región andina y cuatro géneros (Aiphanes, Ceroxylon, Parajubaea y Wettinia) tienen su centro de distribución en los Andes. Latitudinalmente se tiene la mayor riqueza específica y endemismos en Colombia, decreciendo hacia Venezuela y Bolivia (Borchsenius y Moraes 2006).

En Venezuela, la familia se encuentra ampliamente distribuida a lo largo de todo el territorio continental, con excepción de las dependencias federales insulares, y está representada por 30 géneros y 101 especies autóctonas (Stauffer 1999, Hokche et al. 2008). A pesar de ello, la riqueza y diversidad de elementos autóctonos de Venezuela sigue siendo insuficientemente conocida. El estado Zulia se encuentra entre los principales donde se han colectado palmas, como indica Espinoza (2008) en una revisión de la colección de Arecaceae del Herbario Nacional de Venezuela (VEN). Sin embargo, se carece de información sobre áreas de esta región que han sido insuficientemente estudiadas por su accidentada geografía y/o compleja situación geopolítica y que pueden concentrar gran riqueza y diversidad de palmeras como ocurre en la serranía de Perijá, que constituye el ramal más septentrional de la Cordillera de Los Andes, fronterizo entre Venezuela y Colombia.
El presente trabajo tiene por objetivo describir algunos aspectos florísticos y ecológicos a nivel de hábitat, distribución y observaciones sobre el estatus de conservación y amenazas a las poblaciones de siete especies de palmeras: Chamaedorea linearis, $C$. pinnatifrons, Geonoma interrupta, G. undata, Hyospathe pittieri, Prestoea acuminata y Wettinia praemorsa presentes en bosques nublados de la vertiente noreste de la serranía de Perijá, estado Zulia.

\section{Metodología}

Situación geográfica y área de estudio. La serranía de Perijá es una cadena montañosa ubicada en las estribaciones septentrionales de la Cordillera Oriental de Los Andes, formando parte de la frontera internacional entre Venezuela y Colombia. Esta es considerada uno de los centros de endemismo y refugios pleistocénicos de mayor importancia para el conocimiento de la historia natural de los ecosistemas del norte de Suramérica (Hooghiemstra y Cleef 1995). Esta zona ha sido objeto de una acelerada deforestación para la explotación de numerosos recursos naturales, desde el piedemonte hasta algunos sitios de alta montaña. La perdida de la cobertura vegetal original y la fragmentación de hábitats representan las principales amenazas sobre diversas poblaciones de organismos.

El área de estudio se localiza en la vertiente noreste de la serranía de Perijá, entre los $10^{\circ} 20^{\prime} \mathrm{N}$ y $072^{\circ} 33^{\prime} \mathrm{O}$, entre los 1100 y $1930 \mathrm{msnm}$, en los sectores de Cerro Las Antenas y Lajas, que conforman una divisoria de aguas natural entre los ríos Macoita, Piché y Lajas, en el municipio Rosario de Perijá, estado Zulia, al noroeste de Venezuela (Figura 1).

Fases de campoy de laboratorio. Entre los meses de mayo de 2009 y agosto de 2010 se realizaron diferentes recorridos en las localidades de Cerro las Antenas y Lajas, donde se colectó material correspondiente a la familia Arecaceae. Se tomaron muestras vegetativas y reproductivas correspondientes a cada espécimen; cada colección estuvo acompañada de fotografías del espécimen en su hábitat y anotaciones sobre la distribución, densidades, abundancia de individuos, frecuencia y etapa fenológica de desarrollo. Además, durante los recorridos de campo se anotaron observaciones sobre los hábitats de las diferentes especies de palmeras estudiadas, con 
base en características fisiográficas locales (relieve y pendiente de suelos) y de integridad biótica de la cobertura forestal (grado de naturalidad o de intervención dentro del bosque).

Las muestras colectadas en campo fueron herborizadas y depositadas en el Herbario del Museo de Biología de la Universidad del Zulia (HMBLUZ) adscrito a la Facultad Experimental de Ciencias de la Universidad del Zulia (LUZ) en Maracaibo, estado Zulia. Se realizaron determinaciones por medio de claves taxonómicas y comparación con material depositado en el Herbario Nacional de Venezuela (VEN) en Caracas, Herbario del Zulia (HERZU), Herbario de Coro (CORO) en el estado Falcón y el Herbario del Museo de Biología de la Universidad del Zulia (HMBLUZ). También se verificaron especímenes de los herbarios digitales del Missouri Botanical Garden (MOBOT) y Field Museum de Chicago. La información se tomó de los ejemplares y de literatura especializada.

\section{Resultados y discusión}

Se describen a continuación las especies Chamaedorea linearis, C. pinnatifrons, Geonoma interrupta, G. undata, Hyospathe pittieri, Prestoea acuminata y Wettinia praemorsa, así como diferentes aspectos

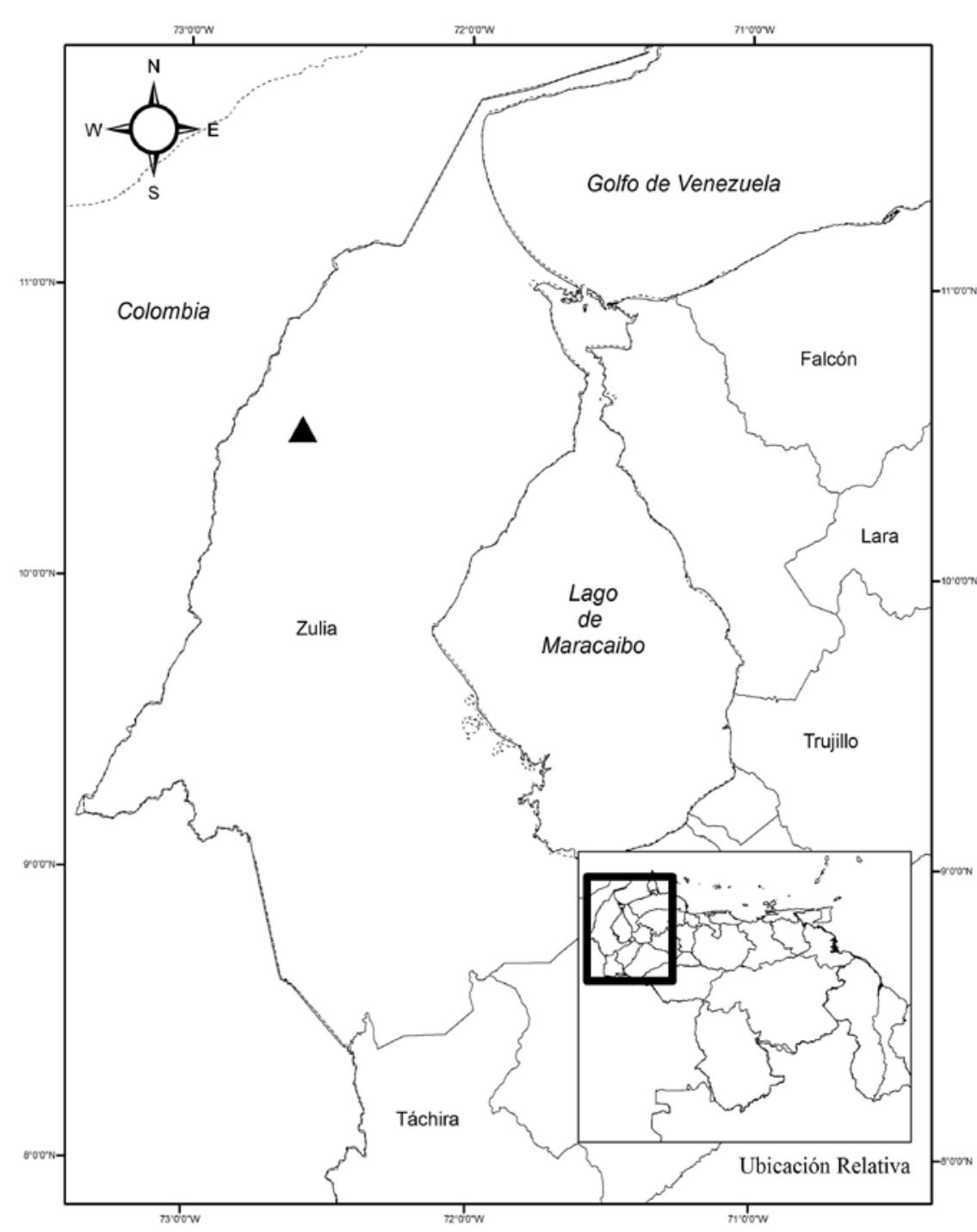

Figura 1. Ubicación geográfica del estado Zulia, Venezuela.

ecológicos a nivel de su hábitat, distribución y otras observaciones sobre sus poblaciones presentes en la vertiente noreste de la serranía de Perijá, estado Zulia.

\section{Chamaedorea linearis (Ruiz \& Pav.) Mart.}

Palmera solitaria, dioica, de porte mediano de 3 a $7 \mathrm{~m}$ de alto; tallo erecto, delgado, de hasta $10 \mathrm{~cm}$ de perímetro, marcadamente anillado y de color verde o verde amarillento. Corona formada por 3-8 hojas pinnadas (11-62 pinnas), las terminales ligeramente sigmoideas, las laterales lanceoladas (16-45 $\mathrm{cm}$ de largo por 2,5-12 $\mathrm{cm}$ de ancho) con el ápice acuminado. Vaina muy desarrollada; pecíolo $30-37 \mathrm{~cm}$, raquis $60-120 \mathrm{~cm}$. Inflorescencia en racimos erectos, originadas por debajo del punto de inserción de las hojas, las masculinas 3-18 por nudo y las femeninas solitarias, ambas con 5-53 ejes florales, brácteas pedunculares cortas. Flores pequeñas unisexuales, de color amarillento. Fruto drupa globosa de 0,8-2,5 cm de diámetro, de color rojo al madurar (Figuras 2 y 3 ). 


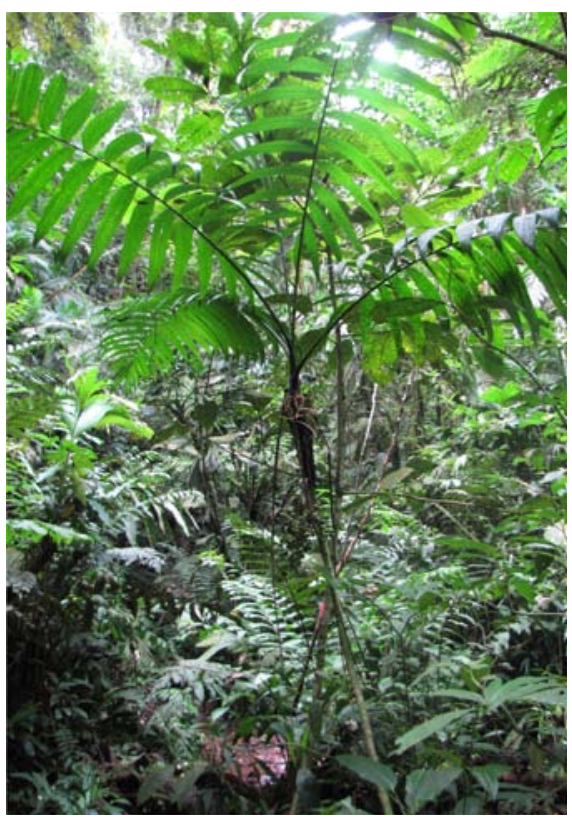

Figura 2. Individuo femenino de Chamaedorea linearis (Ruiz \& Pav.) Mart.

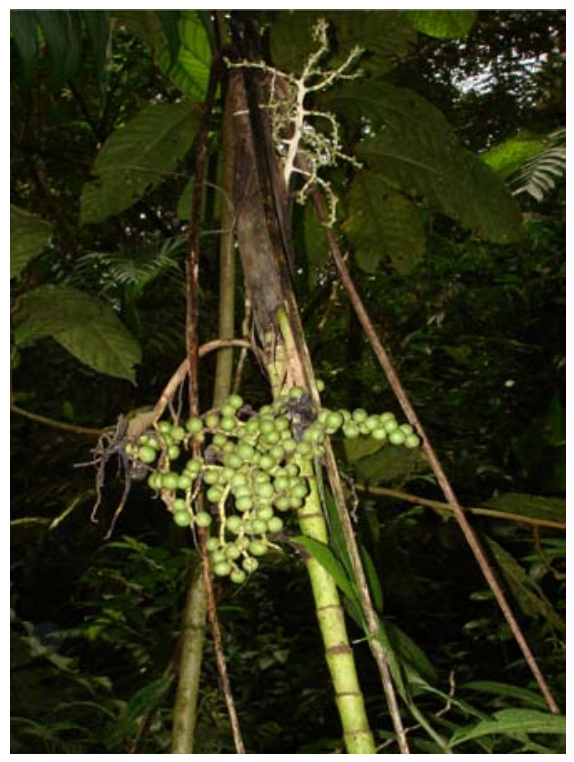

Figura 3. Frutos de Chamaedorea linearis (Ruiz \& Pav.) Mart.

Ejemplares: ZULIA. Mpio. Rosario de Perijá, Cerro Las Antenas, $1450 \mathrm{msnm}, 25 / 06 / 2009$, Arias, J. C. 390, 398 (HMBLUZ); 27/06/2009, 395 (HMBLUZ). Hábitat local: Bosques húmedos montanos, altos, densos a medio-densos, naturales o semiintervenidos (con poca perturbación, como algunas extracciones de árbo-

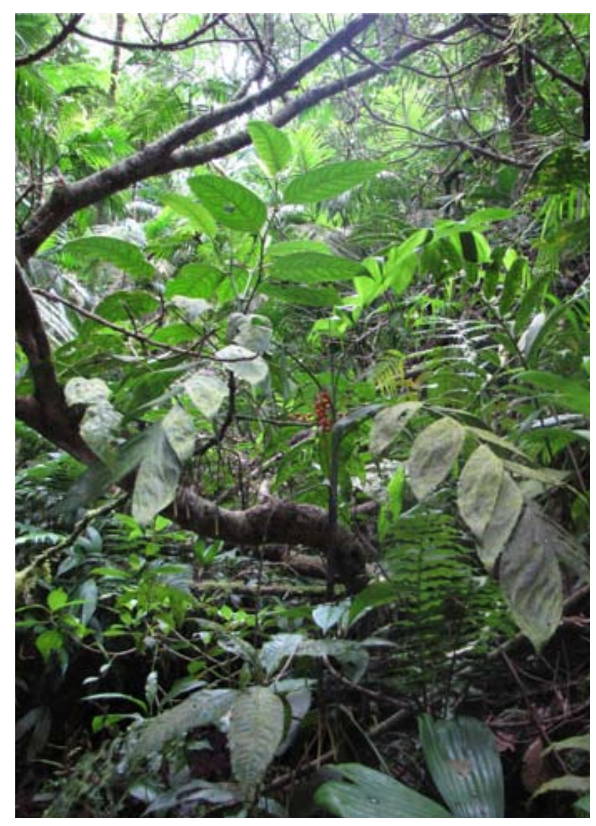

Figura 4. Individuo femenino de

Chamaedorea pinnatifrons (Jacq.) Oerst.

les maderables), sobre todo en zonas cercanas a quebradas entre los 1280 y 1700 msnm. Distribución en Venezuela: Falcón, Lara, Mérida y Zulia, creciendo en el bosque nublado y subpáramo, entre los 1200$2700 \mathrm{msnm}$ (Llamozas et al. 2003). Observación: Nuevo registro para el estado Zulia.

Chamaedorea pinnatifrons (Jacq.) Oerst.

Palmera solitaria, dioica, de 0,80 a $2 \mathrm{~m}$ de alto; tronco erecto a decumbente, perímetro de unos 1,50-6 $\mathrm{cm}$, con marcadas cicatrices foliares. En la base cubierta de raíces aéreas. Hojas de 3 a 6, ligeramente arqueadas; vainas hasta $25 \mathrm{~cm}$ de largo; pecíolos hasta $19 \mathrm{~cm}$ de largo, casi redondeados; raquis de 30 a $40 \mathrm{~cm}$ de largo, con 6 a 8 pares de pinnas, alternas u opuestas, sigmoideas, de 30 a $37 \mathrm{~cm}$ de largo por 4 a $7 \mathrm{~cm}$ de ancho, de color verde puro; las pinnas terminales concrescentes. Inflorescencias axilares; las masculinas péndulas y las femeninas escasas y casi erectas. Frutos ovoides, de color amarillo-anaranjado, de 0,5 a 1,5 cm de largo y $6 \mathrm{~mm}$ de diámetro; al madurar toma una coloración negruzca (Figura 4).

Ejemplares: ZULIA. Mpio. Rosario de Perijá, Cerro Las Antenas, $1450 \mathrm{msnm}, 28 / \mathrm{V} / 2009$, Arias, J. C. 388, 27/VI/2009; 393 (HMBLUZ). Hábitat local: Bosques húmedos montanos, medios a altos, medio-densos, naturales a intervenidos (con perturbaciones pocas a intermedias como pequeños claros) entre los 1200 y 1900 msnm, formando parte del estrato inferior, principalmente en laderas de poca pendiente. Distribución en Venezuela: Anzoátegui, Aragua, Cojedes, Distrito Federal, Falcón, Lara, Mérida, Miranda, Portuguesa, Táchira, Trujillo, Yaracuy, Zulia. Observación: Presenta una amplia distribución en la zona.

Geonoma interrupta (Ruiz \& Pav.) Mart.

Palmera solitaria o multicaule, monoica; de 2 a $5 \mathrm{~m}$ de alto y 6 a $9 \mathrm{~cm}$ de perímetro. Pecíolos más allá de la vaina de 30 a $60 \mathrm{~cm}$ de largo. Hojas pinnadas en número de 4 a 10, arqueadas, de color verde oscuro. Vainas de las hojas abiertas, hasta $1 \mathrm{~m}$ de largo, de color morado; raquis de 120 a $200 \mathrm{~cm}$ de largo con 18-35 pares de pinnas, las centrales de $48 \mathrm{~cm}$ de largo y $5 \mathrm{~cm}$ de ancho, lanceoladas y multinervadas. Inflorescencias infrafoliares, paniculadoramificadas; pedúnculos de 20 a $50 \mathrm{~cm}$ de largo, con 60 a 80 ramas 
divididas y colgantes, pubescentes con tricomas simples o estrellados. Flores de color lila; las masculinas cerca de 2,5 a 3 $\mathrm{mm}$; las femeninas de 2 a 2,5 $\mathrm{mm}$; tubo estaminodial subtruncado. Frutos globosos de 0,40 a $1 \mathrm{~cm}$ de diámetro, más o menos lisos y subglobosos a ovoides, de color negro al madurar (Figura 5).

Ejemplares: ZULIA. Mpio. Rosario de Perijá, Cerro Las Antenas, 1682 msnm, Arias, J. C. 396, 397 (HMBLUZ). Hábitat local: Bosques húmedos montanos, medios a altos, medio-densos, naturales a intervenidos (con perturbaciones pocas a intermedias como bordes de caminos y pequeños claros), entre los 1150 y $1800 \mathrm{msnm}$, formando parte del estrato inferior, principalmente en laderas de poca pendiente. Distribución en Venezuela: Anzoátegui, Aragua, Carabobo, Cojedes, Distrito Federal, Falcón, Miranda, Nueva Esparta, Sucre, Táchira, Yaracuy y Zulia creciendo en el bosque nublado entre 1300 y $2895 \mathrm{msnm}$ (Llamozas et al. 2003). Observación: individuos de la población en Cerro Las Antenas y Lajas suelen presentar crecimiento monocaule, a diferencia de otras poblaciones en Venezuela.

Geonoma undata Klotzsch.

Palmera solitaria, monoica, de unos $6 \mathrm{~m}$ de altura; tallo erecto de unos $10 \mathrm{~cm}$ de perímetro, de color café claro. Corona formada por 8 hojas pinnadas, de 1,4 - 2,5 $\mathrm{m}$ de largo, vaina de las hojas bien desarrollada y de color rojo; hojas con 10-12 pares de pinnas, irregular o casi regularmente divididas, anchas o estrechas, abiertas en el mismo plano, a veces algunas en distintos planos y con el ápice péndulo, dando a la hoja una apariencia plumosa. Inflorescencia paniculada, originada por debajo de las hojas, ramificada en 2 o 3 órdenes, pedúnculo $15-32 \mathrm{~cm}$, raquis 54-69 cm, con 11-24 ramificaciones primarias; ejes florales 12$47 \mathrm{~cm}$ de largo, verdes o rojizos en fruto. Flores pequeñas unisexuales, en grupos de 3 con una femenina en el centro y dos masculinas laterales. Fruto drupa ovoide apiculada en el ápice, 8-9 mm de largo por 6-7 $\mathrm{mm}$ de ancho, de color negro al madu$\operatorname{rar}$ (Figuras 6 y 7 ).

Ejemplares: ZULIA. Serranía de Perijá, Cerro Las Antenas, $1500 \mathrm{msnm}, 25-\mathrm{VI}-2009$; J.C. Arias 389 (HMBLUZ). Hábitat local: Bosques húmedos montanos, altos, densos a medio-densos, naturales o semi-intervenidos (con poca perturbación, como algunas extracciones de árboles maderables). Distribución en Venezuela: Aragua, Barinas, Carabobo, Distrito Federal, Falcón, Mérida, Miranda, Portuguesa, Táchira, Trujillo y Zulia, creciendo en el bosque nublado entre 1300 y $2895 \mathrm{msnm}$ (Llamozas et al. 2003). Observaciones: Nuevo registro para el estado Zulia. Solo 10 individuos fueron hallados a $1540 \mathrm{msnm}$, expuesto a orillas de intersección entre carretera que comunica al sector Lajas con Cerro Las Antenas.

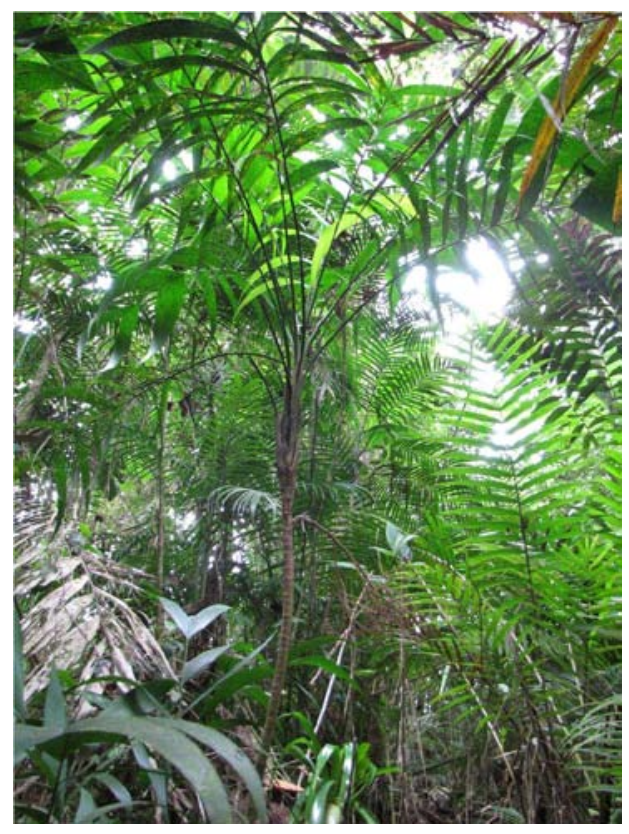

Figura 5. Ejemplar de Geonoma interrupta (Ruiz \& Pav.) Mart.

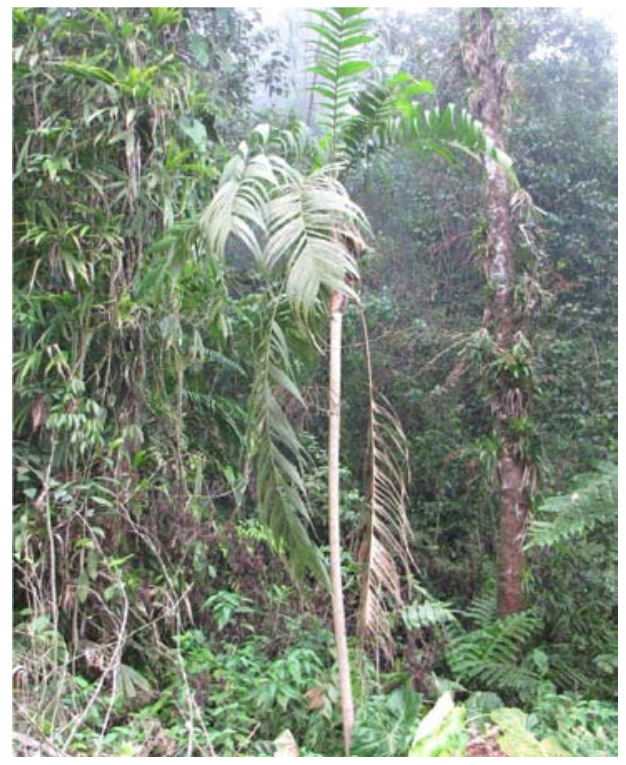

Figura 6. Individuo de Geonoma undata Klotzsch

\section{Hyospathe pittieri Burret}

Palmera multicaule, hasta con 15 tallos juntos, parduzcos, que van desde los 3 hasta $\operatorname{los} 12 \mathrm{~m}$ de alto, con $5-10 \mathrm{~cm}$ de perímetro. Hojas regularmente pinnadas de 5 a 7, vaina de $30 \mathrm{~cm}$ de longitud; raquis de unos $80 \mathrm{~cm}$ longitud, con 22 


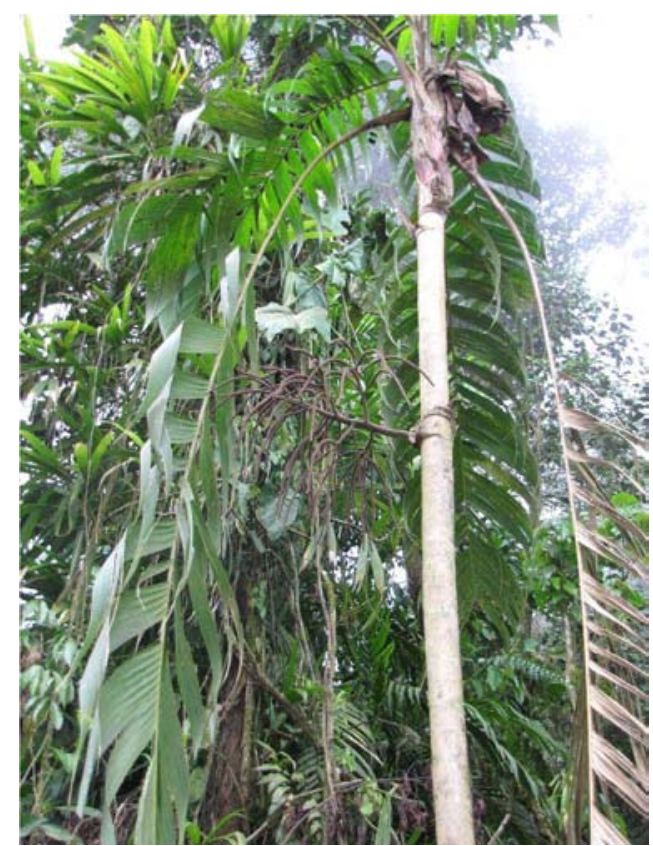

Figura 7. Inflorescencia de Geonoma undata Klotzsch

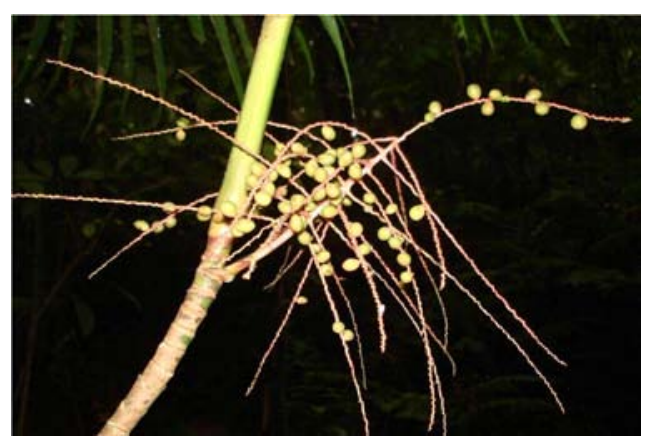

Figura 8. Frutos de Hyospathe pittieri Burret

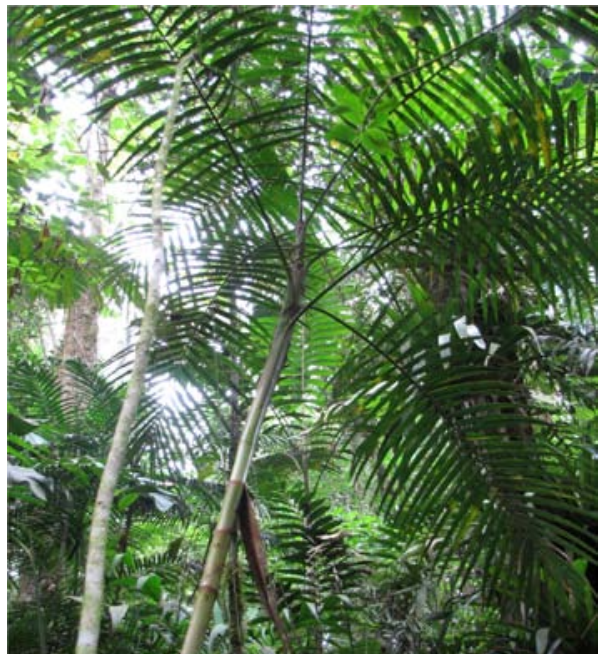

Figura 9. Individuo vegetativo de Prestoea acuminata (Willd.) H.E.Moore pinnas a cada lado, regularmente dispuestas, pátulas. Inflorescencias que nacen por debajo de la vaina foliar, flores en tríadas irregulares, alzadas, sésiles o ligeramente pedunculadas; estaminadas con seis estambres fértiles dispuestos en dos verticilos: tres estambres cortos antisépalos insertos en la base de un pistilodio y tres estambres más alargados antipétalos insertos cerca del vértice del pistiloidio. Infrutescencia infrafoliar rojiza. Frutos verdes cuando inmaduros, negros cuando maduros (Figura 8).

Ejemplares: ZULIA. Mpio. Rosario de Perijá, Cerro Las Antenas, 1450 msnm, 28/V/2009, Arias, J. C. 385; 27/VI/2009, 391, 392 (HMBLUZ). Hábitat local: Bosques húmedos montanos, medios a altos, medio-densos, naturales a intervenidos (con perturbaciones moderadas como bordes de caminos y claros como producto de la tala selectiva), entre los 1200 y $1900 \mathrm{msnm}$, formando parte del estrato inferior. Distribución en Venezuela: Amazonas, Aragua, Bolívar, Cojedes, Lara, Táchira, Yaracuy y Zulia con base en los reportes catalogados como H. elegans en Hokche et al. (2008). Observación: Es la especie más abundante para las localidades de Cerro Las Antenas y Lajas.

\section{Prestoea acuminata (Willd.) H.E. Moore.}

Palmera con tallos solitarios o múltiples (3-5), 3-8 m y 5.5-32 cm de perímetro. Raíces adventicias con pequeñas espinas. Pecíolos 10-60 cm, la vaina cerrada hasta formar un pseudocaule de 40-100 cm, verde a (con mayor frecuencia) castaño o morado. Láminas foliares con cerca de 1,9-3 m, las pinnas 33-59 por lado, 17-95 por 1-8 cm (las del medio). Inflorescencias infrafoliares, racemosamente ramificadas; profilo $28-70 \mathrm{~cm}$; pedúnculo con $8-17 \mathrm{~cm}$, con la bráctea de $87-150 \mathrm{~cm}$; raquis $48-89 \mathrm{~cm}$; raquilas $43-125$, glabrescentes a inconspicuamente pubescentes con pelos hasta $0,1 \mathrm{~mm}$, más o menos dendríticos o subestrellados, como escamas. Flores masculinas 3,5-7 mm, rosadas, los pétalos subglabros o diminutamente puberulentos abaxialmente. Flores femeninas con 2,5-4,5 mm, rosadas. Frutos son de forma ovoide, de color negro de $12 \mathrm{~mm}$ aproximadamente (Figura 9).

Ejemplares: ZULIA. Mpio. Rosario de Perijá, Cerro Las Antenas, 1600 msnm, 27/06/2009, Arias, J. 399 (HMBLUZ). Hábitat local: Bosques húmedos montanos, medios a altos, medio-densos, naturales a intervenidos (con perturbaciones moderadas como bordes de caminos y claros, producto de la tala selectiva) entre los 1100 y $1900 \mathrm{msnm}$, formando parte del estrato inferior. Distribución en Venezuela: Aragua, Distrito Federal, Falcón, Mérida, Miranda, Monagas, Sucre, Táchira, Trujillo y Yaracuy creciendo en el bosque nublado entre 600 y 2000 msnm (Llamozas et al. 2003, Hokche et al. 2008). Observación: En los sectores de Lajas y Cerro Las 
Antenas es menos frecuente por encima de los 1300 msnm.

Wettinia praemorsa (Willd.) Wess. Boer.

Palmera solitaria o multicaule, monoica, inerme, de 10 a $15 \mathrm{~m}$ de alto. Tronco cilíndrico, liso, de 8 a $40 \mathrm{~cm}$ de perímetro, con raíces aéreas hasta $40 \mathrm{~cm}$ de largo, a menudo cubierto de numerosos líquenes. Pocas hojas, por lo general 3 o 4, de 2 a 2,50 m de largo, con 25 a 30 pares de pinnas; las terminales ordinariamente forman un abanico a medio abrir, las otras tienen forma lanceoladas a deltoides en la base, con ápice redondeado y márgenes premorsas; suelen medir de 20 a $40 \mathrm{~cm}$ de largo por 5 a $10 \mathrm{~cm}$ de ancho. Brácteas rodeando las inflorescencias pequeñas, de 10 a $30 \mathrm{~cm}$ de largo. Inflorescencias de 15 a $20 \mathrm{~cm}$ de largo dispuestas en grupos sobre el tronco y debajo de las hojas, protegidas por una bráctea (espata) color marrón. Pedicelo de inflorescencia con tricomas color negro. Frutos de unos 4,5 cm de largo por $3 \mathrm{~cm}$ de ancho, de color verde al madurar (Figura 10).

Ejemplares: ZULIA. Mpio. Rosario de Perijá, Cerro Las Antenas, 1450 msnm, 28/V/2009, Arias, J. C. 387 (HMBLUZ). Hábitat local: Bosques húmedos montanos, medios a altos, medio-densos, naturales a intervenidos (con perturbaciones moderadas como bordes de caminos y claros como producto de la tala selectiva) entre los 1400 y $1930 \mathrm{msnm}$, en cumbres y laderas superiores de pendientes pronunciadas. Distribución en Venezuela: Aragua, Carabobo, Cojedes, Distrito Federal, Falcón, Lara, Mérida, Miranda, Táchira, Trujillo, Yaracuy y Zulia en el bosque nublado entre los 1000 y $2200 \mathrm{msnm}$ (Llamozas et al. 2003, Hokche et al. 2008). Observaciones: Abundante en localidades de la cuenca alta del río Lajas, por encima de los $1400 \mathrm{msnm}$.

Observaciones en relación con el estatus de conservación y amenazas a las poblaciones de palmeras de bosque nublado de la vertiente noreste de la serranía de Perijá, Venezuela:

En Venezuela se ha reportado que la principal amenaza a la que se enfrentan las poblaciones de $C$. linearis, C. pinnatifrons, G. interrupta, G. undata, $H$. pittieri, $P$. acuminata y $W$. praemorsa se debe a la destrucción de su hábitat para el desarrollo de actividades agrícolas y urbanísticas (Stauffer en Llamozas et al. 2003).

Actualmente la tala y quema de los bosques

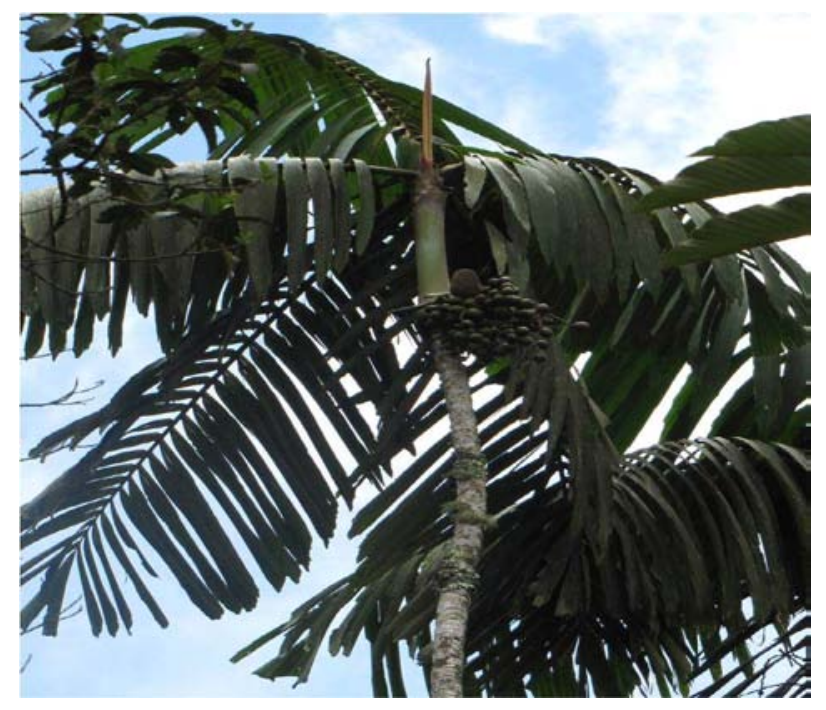

Figura 10. Individuo con frutos de Wettinia praemorsa (Willd.) Wess. Boer

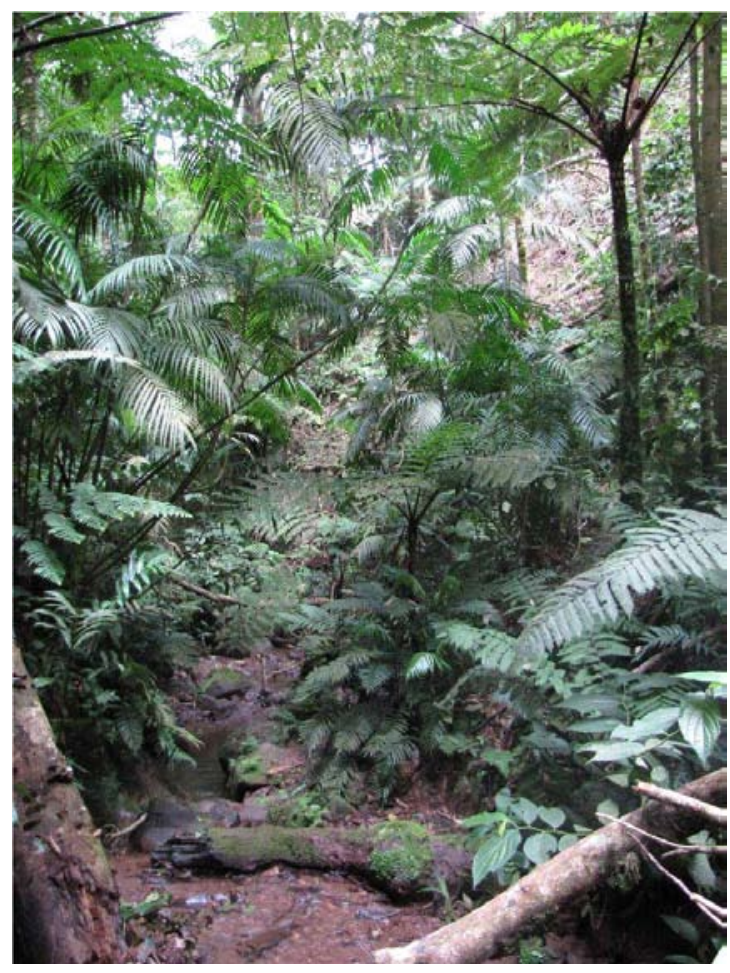

Figura 11. Bosque ribereño de H. pittieri y C. linearis.

montanos de la serranía de Perijá está causando severos impactos sobre la cobertura vegetal, generando extensos procesos erosivos y de sedimentación de las cuencas hidrográficas, como producto de la fuerte presión de uso de las tierras para el desarrollo agrícola incontrolado de la cuenca del río Lajas y otras áreas de la vertiente noreste de esta cordillera. 
Estas tendencias actuales de las actividades agrícolas implican la simplificación del paisaje y la reducción de la diversidad biológica, que alcanzan una forma extrema con la expansión de monocultivos (Altieri 1996) como el de la arácea Xanthosoma sagittifolium (L.) Schott, vulgarmente conocida como Malanga, cuyos tubérculos son comercializados en la región.

La especie $C$. linearis ha sido poco colectada en Venezuela y cuenta con poblaciones muy reducidas en el país (Stauffer en Llamozas et al. 2003) así como localmente en el área de Cerro Las Antenas; esta palmera es más frecuente en sitios húmedos cerca de quebradas montanas entre los 1280 y $1600 \mathrm{msnm}$. En el caso de C. pinnatifrons, la población local de Cerro Las Antenas presenta una considerable abundancia y amplia distribución altitudinal comprendida entre los 1200 y $1800 \mathrm{msnm}$, aunque posiblemente menos abundante que en otras partes de Venezuela. Al ser un género dioico, la biología reproductiva y dinámica poblacional de las especies de Chamaedorea es más sensible ante posibles perturbaciones.

G. interrupta parece ser más abundante en sitios donde la cobertura forestal permite cierta entrada de luz, ya sea por la existencia de pequeños claros en el bosque o por el grado de exposición natural a la luz del sotobosque. Esta especie presenta una amplia distribución altitudinal, comprendida entre los 1150 y $1600 \mathrm{msnm}$. Los individuos suelen presentar crecimiento monocaule, a diferencia de otras poblaciones venezolanas de G. interrupta. Para G. undata solo 2 individuos fueron hallados en el área estudiada. A pesar de realizarse una serie de recorridos exhaustivos, no se encontró más representantes de este taxón en la zona, lo cual pone en evidencia el estado crítico de esta especie en el área estudiada. Si bien existen poblaciones protegidas de G. undata en los Parques Nacionales Juan Crisóstomo Falcón y Yacambú (Stauffer en Llamozas et al. 2003, Mondragón et al. 2008) es de importancia crítica establecer un control sobre las amenazas a la población local de $G$. undata en las localidades de Lajas y Cerro Las Antenas, donde no existe una figura de Área Natural Protegida.

H. pittieri presenta la población más importante en términos de densidad y una amplia distribución altitudinal comprendida entre los 1150 y 1600 msnm, con individuos que pueden presentar hasta 14 troncos. Cabe destacar la amplitud de condiciones en las que esta palmera se puede desarrollar dentro del bosque húmedo, ya sea cerca de quebradas (Figura 11), en terrazas o laderas de acusada pendiente, bajo condiciones naturales o frente a ligeras perturbaciones antrópicas como la apertura de claros (por la tala) o de pequeños caminos.

$P$. acuminata se encuentra protegida en algunas zonas del país como el Parque Nacional Henri Pittier y en el Parque Nacional El Ávila (Stauffer en Llamozas et al. 2003), sin embargo la población local de Cerro Las Antenas y Lajas se encuentra muy reducida debido a la tala y quema de los bosques donde naturalmente se desarrolla esta especie. Actualmente la frontera agrícola se ha extendido hasta los $1100 \mathrm{msnm}$ en algunos sectores de la vertiente noreste de Perijá, mientras esta especie presenta una distribución altitudinal aproximada entre los 8001300 msnm en el área de la cuenca del Lajas; en vista de esta situación sería pertinente catalogar a esta población en estado crítico de amenaza, siendo necesario estudiar su biología in situ para su conservación y restauración ecológica.

$W$. praemorsa presenta una población relativamente grande en el sector superior de la cuenca del río Lajas, siendo abundante en laderas superiores sobre suelos orgánicos bien drenados, con una distribución altitudinal entre los 1400 y $1930 \mathrm{msnm}$ en el sector Cerro Las Antenas. Se puede desarrollar tanto en bosques primarios como secundarios por lo cual sería de especial utilidad estudiar sus atributos biológicos con miras a la restauración ecológica local de bosques nublados sobre pendientes pronunciadas.

Las poblaciones locales de palmeras (Arecaceae) presentes en la cuenca del río Lajas-Cerro Las Antenas, se encuentran amenazadas por la fragmentación y destrucción de su hábitat natural debido a la deforestación de los bosques nublados. Especial atención merecen las poblaciones de G. undata y $P$. acuminata que se encontrarían en estado crítico de amenaza, mientras que $C$. linearis podría considerarse como vulnerable, debido a que presenta una distribución restringida.

La importancia de las palmeras como elementos clave para el sostenimiento de la biodiversidad de los bosques nublados de la serranía de Perijá y la 
actual situación de amenaza bajo la cual se encuentran estas especies, se suman a un conjunto de problemas ecológicos que por la magnitud de su impacto se pueden catalogar como prioritarios para la región zuliana, por lo cual requieren de un tratamiento prioritario.

El estudio y manejo sistemático de estas especies permitirá llevar a cabo con éxito planes de conservación y restauración ecológica. Es necesario realizar estudios de la biología de estas palmeras in situ así como planificar y desarrollar la formación y articulación de recursos humanos locales e instituciones con competencia en la región para la ejecución de planes de manejo y conservación viables a corto, mediano y a largo plazo.

\section{Agradecimientos}

A la fundación Provita, por su apoyo a la investigación en conservación de palmeras amenazadas, a través del proyecto $\mathrm{N}^{\circ} 2011-19$ de la Iniciativa de Especies Amenazadas. A Natanael MoralesSzymanski, Fabián Rojas-Sáenz y Andreiz PaterninaBaleta por su ayuda en el trabajo de campo. A Rita E. Fereira-Hung por la revisión crítica de este documento. A Miguel Pietrangeli por su colaboración en el herbario HERZU. Un agradecimiento especial a José Ramón Socorro, por la colaboración brindada en Cerro Las Antenas, a Vicente y a Rafael y su familia en la estación de K'lor FM, Municipio Rosario de Perijá. Dos revisores anónimos aportaron valiosas sugerencias en la redacción de este trabajo.

\section{Literatura citada}

Altieri M. 1996. El rol ecológico de la biodiversidad en agroecosistemas. En: Agroecología y agricultura sostenible. Diseño y manejo de sistemas agrícolas sostenibles. La Habana: CLADES, CEAS-ISCAH. 84 pp.

Balick MJ, Beck H. 1990. Useful palms of world: a synoptic bibliography. New York: Columbia University Press. 794 pp.

Balslev H, Barfod A. 1987. Ecuadorean palms: An overview. Opera Bot. 92: 17-35.
Balslev H, Grandez C, Paniagua-Zambrana N, Møller AL, Lykke-Hansen S. 2008. Palmas (Arecaceae) útiles en los alrededores de Iquitos, Amazonía Peruana. Rev Peru Biol. 15 (Supl. 1): 121-32.

Borchsenius F, Moraes M. 2006. Diversidad y usos de palmeras andinas (Arecaceae). La Paz: Botánica Económica de los Andes. p. 412-33.

Cabrera H, Wallace R. 2007. Densidad y distribución espacial de palmeras arborescentes en un bosque preandinoamazónico de Bolivia. Ecol Bol. 42 (2):121-35.

Dransfield J, Uhl N, Asmussen C, Baker W, Harley M, Lewis C. 2008. Genera Palmarum. The evolution and classification of palms. Richmond: Royal Botanic Gardens. 610 pp.

Espinoza Y. 2008. Estado actual de la colección de palmas (Arecaceae) del Herbario Nacional de Venezuela (VEN). Rev Peru Biol. 15 (Supl. 1): 97-101.

Galeano G, Bernal R. 2010. Palmas de Colombia. Guía de campo. Bogotá: Editorial Universidad Nacional de Colombia. 688 pp.

Henderson A. 2004. A multivariate analysis of Hyospathe (Palmae). Am J Bot. 91: 953-65.

Hokche O, Berry P, Huber O. (eds.). 2008. Nuevo catálogo de la flora vascular de Venezuela. Caracas: Fundación Instituto Botánico de Venezuela «Dr. T. Lasser». 859 pp.

Hooghiemstra H, Cleef A. 1995. Pleistocene climatic change and environmental and generic dynamics in the north Andean montane forest and paramo. In: Churchill SP et al. (eds.). Biodiversity and conservation of neotropical montane forests. New York: The New York Botanical Garden. p. 35-49.

Hoyos J, Braun A. 2001. Palmas en Venezuela. Caracas: Sociedad de Ciencias Naturales La Salle. 424 pp.

Llamozas S, Duno de Stefano R, Meier W, et al. 2003. Libro rojo de la flora venezolana. Caracas: Provita, Fundación Polar e Instituto Botánico de Venezuela. 533 pp.

Mondragón A, Álvarez L, Alvarado H, Dávila M. 2008. Primer reporte de Geonoma undata Klotzsch (Arecaceae) en el Estado Lara, Venezuela. Bol Cen Invest Biol. 42 (2): 27580.

Pintaud J-C, Galeano G, Balslev H, et al. 2008. Las palmeras de América del Sur: diversidad, distribución e historia evolutiva. Rev Peru Biol. 15: 7-29.

Scariot A. 1999. Forest fragmentation effects on palm diversity in central Amazonia. J Ecol. 87: 66-76.

Stauffer F. 1999 Datos preliminares para la actualización de la flora de palmas (Arecaceae) de Venezuela. Acta Bot Venezuel. 22 (1): 77-107.

Uhl N, Dransûeld J. 1987. Genera Palmarum: a classiûcation of palms based on the work of H. E. Moore Jr. Lawrence. Kansas: International Palm Society and L. H. Bailey Hortorium. 610 pp. 